Verification Test Suite for

Physics Simulation Codes

J. S. Brock, J. R. Kamm, W. J. Rider, S. Brandon,

C. Woodward, P. Knupp, T. G. Trucano

December 22, 2006 
This document was prepared as an account of work sponsored by an agency of the United States Government. Neither the United States Government nor the University of California nor any of their employees, makes any warranty, express or implied, or assumes any legal liability or responsibility for the accuracy, completeness, or usefulness of any information, apparatus, product, or process disclosed, or represents that its use would not infringe privately owned rights. Reference herein to any specific commercial product, process, or service by trade name, trademark, manufacturer, or otherwise, does not necessarily constitute or imply its endorsement, recommendation, or favoring by the United States Government or the University of California. The views and opinions of authors expressed herein do not necessarily state or reflect those of the United States Government or the University of California, and shall not be used for advertising or product endorsement purposes.

This work was performed under the auspices of the U.S. Department of Energy by University of California, Lawrence Livermore National Laboratory under Contract W-7405-Eng-48. 


\title{
Verification Test Suite for Physics Simulation Codes
}

\author{
Jerry S. Brock, James R. Kamm and William J. Rider \\ Los Alamos National Laboratory \\ Scott Brandon, Carol Woodward \\ Lawrence Livermore National Laboratory \\ Patrick Knupp, Timothy G. Trucano \\ Sandia National Laboratory
}

\section{Introduction}

The DOE/NNSA Advanced Simulation \& Computing (ASC) Program directs the development, demonstration and deployment of physics simulation codes. The defensible utilization of these codes for high-consequence decisions requires rigorous verification and validation of the simulation software. The physics and engineering codes used at Los Alamos National Laboratory (LANL), Lawrence Livermore National Laboratory (LLNL), and Sandia National Laboratory (SNL) are arguably among the most complex utilized in computational science. Verification represents an important aspect of the development, assessment and application of simulation software for physics and engineering. The purpose of this note is to formally document the existing tri-laboratory suite of verification problems used by LANL, LLNL, and SNL, i.e., the Tri-Lab Verification Test Suite.

Verification is often referred to as ensuring that "the [discrete] equations are solved [numerically] correctly." More precisely, verification develops evidence of mathematical consistency between continuum partial differential equations (PDEs) and their discrete analogues, and provides an approach by which to estimate discretization errors. There are two variants of verification: (1) code verification, which compares simulation results to known analytical solutions, and (2) calculation verification, which estimates convergence rates and discretization errors without knowledge of a known solution. Together, these verification analyses support defensible verification and validation $(\mathrm{V} \& \mathrm{~V})$ of physics and engineering codes that are used to simulate complex problems that do not possess analytical solutions.

Discretization errors (e.g., spatial and temporal errors) are embedded in the numerical solutions of the PDEs that model the relevant governing equations. Quantifying discretization errors, which comprise only a portion of the total numerical simulation error, is possible through code and calculation verification. Code verification computes the absolute value of discretization errors relative to an exact solution of the governing equations. In contrast, calculation verification, which does not utilize a reference solution, combines an assessment of stable self-convergence and exact solution prediction to quantitatively estimate discretization errors. 
In FY01, representatives of the V\&V programs at LANL, LLNL, and SNL identified a set of verification test problems for the Accelerated Strategic Computing Initiative (ASCI) Program. Specifically, a set of code verification test problems that exercise relevant single- and multiple-physics packages was agreed upon. The verification test suite problems can be evaluated in multidimensional geometry and span both smooth and non-smooth behavior.

\section{Test Problems}

There are currently seven problems in the Tri-Lab Verification Test Suite. The physics modeled in these problems include gas dynamics (Noh and Sedov Problems), coupled gas dynamics and non-linear heat conduction (Reinicke/Meyer-ter-Vehn Problem), coupled gas dynamics and radiation-diffusion (Coggeshall-8 Problem), non-equilibrium radiationdiffusion (Su-Olson Problem), neutron transport (Sood Problem), and high explosives (either the Escape of HE Products or Mader Problem). A brief description of these problems is provided below, followed by a table that catalogues the test problems together with their relevant physics models.

1. Noh Problem [1] Symmetric planar, cylindrical or spherical one-dimensional, inviscid, non-heat conducting, compressible gas dynamics of a polytropic gas, which tests a code's ability to convert kinetic energy into internal energy. This problem admits a closed-form self-similar solution.

2. Sedov Problem [2] Symmetric planar, cylindrical or spherical one-dimensional, inviscid, non-heat conducting, compressible gas dynamics of a polytropic gas, which tests a code's ability to convert internal energy into kinetic energy. This problem admits a closed-form, self-similar solution that requires one numerical quadrature.

3. Reinicke/Meyer-ter-Vehn (RMtV) Problem [3] Extension of the spherically symmetric Sedov problem to include non-linear heat conduction. This selfsimilar problem's solution requires the numerical solution of a non-linear eigenvalue problem in the form of coupled, non-linear ordinary differential equations.

4. Coggeshall-8 (Cog-8) Problem [4] Spherically symmetric, one-dimensional problem that couples inviscid, compressible gas dynamics of a polytropic gas with radiation-diffusion. This problem admits a closed-form solution.

5. Su/Olson Problem [5] Non-equilibrium radiation-diffusion physics problem in one-dimensional, Cartesian (slab) geometry. This problem admits a solution that reduces to numerical quadrature.

6. Sood Problem [6] Neutron transport problem in one-dimensional, Cartesian (slab) geometry. This solution to this problem is given in terms of an analytic eigenvalue and corresponding eigenfunction solution.

7a. Escape of HE Products [7a] A constant-velocity piston interacts with a onedimensional, Cartesian (slab) high explosive with a polytropic gas equation of state initiating an unsupported detonation. The straight-line characteristics of this problem admit a closed-form solution. 
7b. Mader Problem [7b] High explosives problem in one-dimensional, Cartesian (slab) geometry for a material with a polytropic gas equation of state. This problem admits a closed-form solution for the material properties in the rarefaction wave behind a steady detonation.

Table 1. Identification of the ASC Tri-Lab Test Suite problems and their corresponding physics models

\begin{tabular}{|l|c|l|l|l|l|}
\hline $\begin{array}{l}\text { Test } \\
\text { Problem }\end{array}$ & $\begin{array}{l}\text { Gas } \\
\text { Dynamics }\end{array}$ & $\begin{array}{l}\text { Non-Linear } \\
\text { Heat } \\
\text { Conduction }\end{array}$ & $\begin{array}{l}\text { Non-Equilib. } \\
\text { Radiation } \\
\text { Diffusion }\end{array}$ & $\begin{array}{l}\text { Neutron } \\
\text { Transport }\end{array}$ & $\begin{array}{l}\text { High } \\
\text { Explosives }\end{array}$ \\
\hline 1. Noh & $\bullet$ & & & & \\
\hline 2. Sedov & $\bullet$ & & & & \\
\hline 3. RMtV & $\bullet$ & $\bullet$ & & & \\
\hline 4. Cog-8 & $\bullet$ & & $\bullet$ & & \\
\hline 5. Su-Olson & & & $\bullet$ & & \\
\hline 6. Sood & & & & & $\bullet$ \\
\hline 7. HE & $\bullet$ & & & & $\bullet$ \\
\hline
\end{tabular}

\section{References}

1. Noh, W.F., "Errors for Calculations of Strong Shocks Using an Artificial Viscosity and an Artificial Heat-Flux," J. Comp. Phys. 72, pp. 78-120 (1987).

2. Sedov, L.I., Similarity and Dimensional Methods in Mechanics, Academic Press, New York, NY, p. 146 ff. (1959).

3. Reinicke, P., and Meyer-ter-Vehn, J., "The Point Explosion with Heat Conduction," Phys. Fluids A 3, pp. 1807-1818 (1991).

4. Coggeshall, S.V., "Analytic Solutions of Hydrodynamics Equations," Phys. Fluids A 3, pp. 757-769 (1991).

5. Su, B., and Olson, G.L., "Benchmark Results for the Non-Equilibrium Marshak Diffusion Problem, ” J. Quant. Spectrosc. Radiat. Transfer 56, pp. 337-351 (1996).

6. Sood, A., Forster, R.A., and Parsons, D.K., "Analytical Benchmark Test Set for Criticality Code Verification," Prog. Nucl. Energy 42, pp. 55-106 (2003).

7a. Fickett, W., and Rivard, W.C., Test Problems for Hydrocodes, LA-5479 (1974).

7b. Fickett, W., and Davis, W.C., Detonation, University of California Press, Berkeley, CA (1979). 